\title{
Hepatic subcapsular steatosis in a patient with insulin dependent diabetes receiving dialysis
}

\author{
C J Burrows, A W Jones
}

Department of Histopathology, Hope Hospital, Salford, Manchester C J Burrows A W Jones

Correspondence to: Dr C J Burrows, Department of Histopathology, Arrowe Park Hospital, Upton, The Wirral, Merseyside L49 5PE. Accepted for publication 7 September 1993

Figure 1 Rim of steatosis under the liver capsule.

\begin{abstract}
Fatty change confined to the subcapsular region of the liver is rare and has always been associated with continuous ambulatory peritoneal dialysis in insulin dependent diabetes. The case of a 41 year old man who died from the complications of his diabetes is reported.
\end{abstract}

( Clin Pathol 1994;47:274-275)

\section{Case report}

A 41 year old man had been an insulin dependent diabetic since the age of $18 . \mathrm{He}$
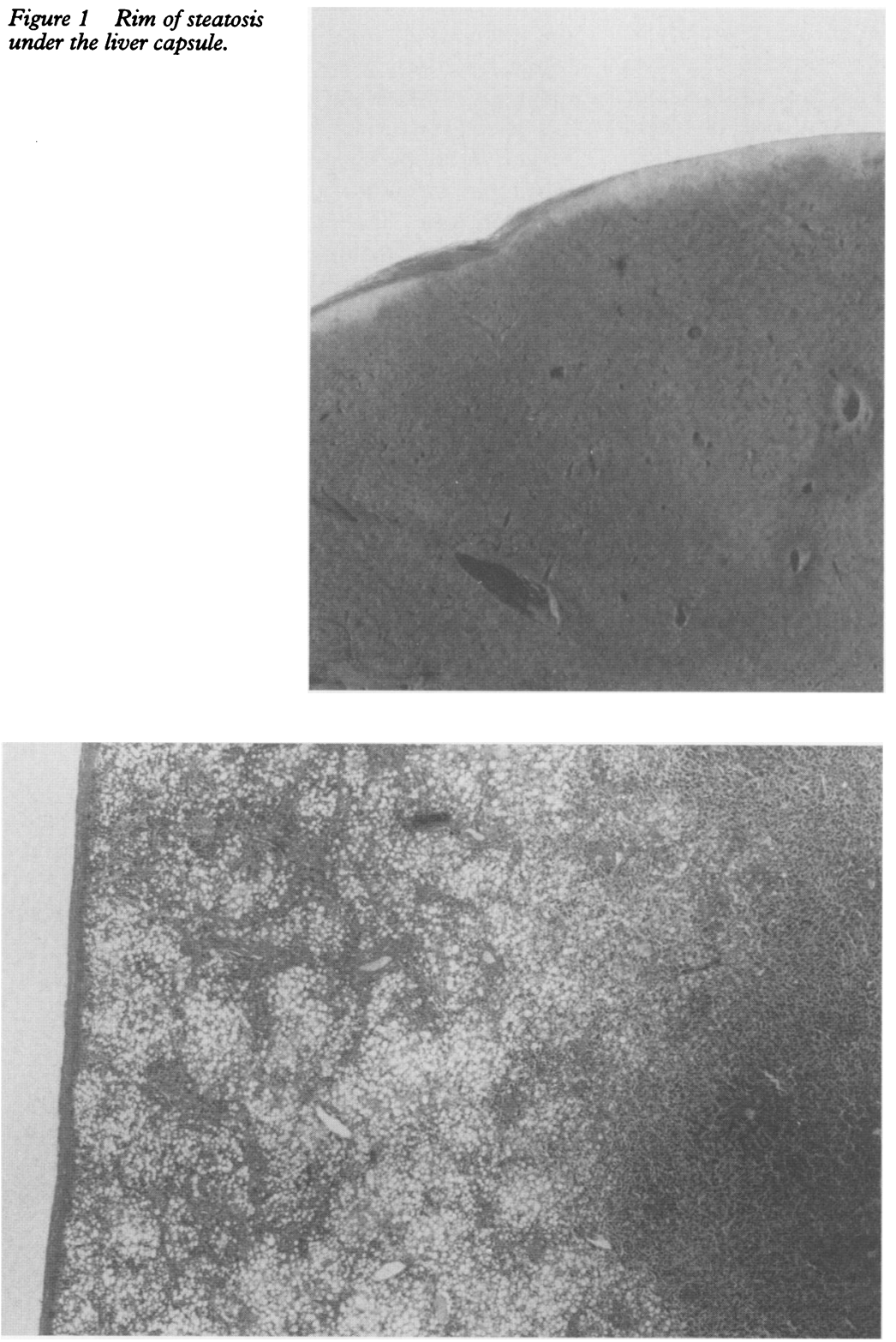

Figure 2 Focal subcapsular steatosis adjacent to normal liver. had retinopathy and neuropathy and had had two myocardial and two cerebral infarctions. Two years before his death he went into chronic renal failure due to diabetic nephropathy and was treated by continuous ambulatory peritoneal dialysis (CAPD) with insulin administered within the dialysis solution.

He had been admitted to the renal unit at Hope Hospital for further education concerning CAPD, when he died following an anterior myocardial infarction. Liver function tests had been within normal limits and he was hepatitis B negative.

A post mortem examination confirmed the recent and previous myocardial and cerebral infarctions. Both kidneys were shrunken and scarred. The liver was of normal size (1650 g), shape, and consistency, but the anterior surface was diffusely yellow with only small areas of normal colouration. On sectioning, this yellow colour was confined to a subcapsular rim of $1.2 \mathrm{~cm}$ in maximum thickness (fig 1). The remainder of the liver looked normal. Microscopic examination showed a well demarcated area of steatosis with the remainder of the liver free of fatty change (fig 2 ).

\section{Discussion}

Fatty change in the liver in most cases is generalised. Subcapsular focal fatty change is rare and is confined to insulin dependent diabetics receiving insulin through CAPD solutions. Ten previous cases have been reported. ${ }^{1}$ This unusual and characteristic pattern of fatty change is of diagnostic and metabolic interest and we describe a further case.

Wanless proposed a mechanism for the formation of subcapsular steatosis affecting the passage of glucose across the liver capsule from the hyperosmotic dialysis solution. ${ }^{2}$ Glucose is absorbed by the hepatocytes, with the subcapsular region absorbing the greatest concentration. The concurrent administration of insulin stimulates glycogen synthesis triglyceride formation and hence steatosis. Due to the high concentration gradient, the subcapsular region is affected preferentially. Subcapsular steatosis is not seen in patients receiving CAPD without intraperitoneal insulin.

Brawer suggested that focal hypoxia was a causative factor in fat deposition. ${ }^{3}$ He looked at a series of focal fatty nodules which seemed to lie at the periphery of the portal venous and hepatic circulations. Focal hypoxia could be a self perpetuating phenomenon in that 
the steatosis would cause swelling of the hepatocytes which could then further compromise the blood supply. This hypothesis is unlikely in this case as over $90 \%$ of the liver capsule was affected.

I am grateful to Dr $S$ Waldeck for permission to report his patient.
1 Grove A, Vyberg B, Vyberg $M$. Focal fatty change of the liver. Virchows Arch (Pathol Anat) 1991;419: 69-75.

2 Wanless I, Bargman J, Oreopoulos D, Vas S. Subcapsular steatonecrosis in response to peritoneal insulin delivery: a clue to the pathogenesis of steatonecrosis in obesity. Mod Pathol 1989;2:69-74.

3 Brawer M, Austin G, Lewin K. Focal fatty change of the liver, a hitherto poorly recognized entity. Gastroenterol 1980;78:247-52.

\title{
Immunohistochemical and in situ hybridisation detection of adenovirus early region 1A (E1A) gene in the microglia of human brain tissue
}

\author{
T Matsuse, Y Namba, K Ikeda, S Inoue, T Hosoi, Y Ouchi, Y Fukuchi, H Orimo
}

Department of

Geriatrics, Faculty of

Medicine, University

of Tokyo

T Matsuse

$S$ Inoue

T Hosoi

Y Ouchi

Y Fukuchi

H Orimo

Department of

Neuropathology,

Institute of Brain

Research, Faculty of

Medicine, University

of Tokyo

Y Namba

Department of

Ultrastructure and

Histochemistry,

Psychiatric Research

Institute of Tokyo

K Ikeda

Correspondence to:

Dr Takeshi Matsuse,

Department of Geriatrics,

Faculty of Medicine,

University of Tokyo, 7-3-1

Hongo Bunkyo-ku, Tokyo

113, Japan.

Accepted for publication

7 September 1993

\begin{abstract}
To investigate whether brain tissue is infected latently by adenovirus via a monocyte/microglia-mediated entry mechanism, brain tissue resected at necropsy from seven senile subjects (five with senile dementia of Alzheimer type (SDAT) and two subjects without pathological changes) was examined for adenovirus early region $1 \mathrm{~A}$ (E1A) gene and its expression using in situ hybridisation and immunohistochemical staining. HLA-DR positive, reactive microglial cells in both SDAT and normal brain tissue showed positive hybridisation and immunoreactive expression of adenovirus E1A. Thus there may be monocyte/ microglia-mediated entry of adenovirus in the central nervous system which would be a novel and presumably common interaction between brain tissue and adenovirus.
\end{abstract}

(F Clin Pathol 1994;47:275-277)

Adenoviruses are common; several of the 41 subtypes of adenovirus cause respiratory infections in man. Group C serotypes $(1,2,5$ and 6) of adenovirus are of particular interest because they are known to reside latently in the tonsils ${ }^{1}$ and peripheral blood mononuclear cells such as lymphocytes ${ }^{2}$ for many years after infection.

The origin of the microglia has long been a subject of debate, but it now seems clear that monocytes enter the normal central nervous system and follow a series of morphological transformations as they differentiate into microglia. As monocytes migrate into the normal central nervous system, they represent potential vehicles for the entry of pathogens into the nervous system. Two members of the lentiviruses, Maedi-Visna (MV) virus and HIV, use the monocyte-mediated ingress mechanism, the so-called "Trojan horse" mechanism of central nervous system invasion, and persistently infect microglia. ${ }^{3}$

The protein products of adenovirus early region $1 \mathrm{~A}(\mathrm{E} 1 \mathrm{~A})$ gene have a role in the activation of other early promotors, as well as transcription from several host promotors. Furthermore, the E1A proteins are essential for the transformation of cells by adenovirus. ${ }^{4}$

The purpose of this study was to examine human senile brain tissues to determine whether those microglia are infected latently by adenovirus. We particularly used senile dementia of Alzheimer type (SDAT) brain tissues because there are abundant reactive microglial cells in the brains of those patients. $^{5}$

\section{Methods}

Five post mortem human brains from patients with a clinicohistopathological diagnosis of SDAT (age 73-93 years, mean 85) and two senile brains without any pathological changes (patients aged 77 and 76 years) were investigated.

Brain tissue obtained at necropsy was snap frozen and stored at $-80^{\circ} \mathrm{C}$. Cryostat sections were fixed in cold acetone and subjected to a combination of in situ hybridisation with digoxigenine-11-dUTP-labelled probe for the E1A viral genome and immunohistochemical staining with HLA-DR monoclonal antibody (two SDAT and one normal brains). Briefly, sections were mounted on coated glass slides and acetylated. The sections were incubated with a hybridisation mixture containing digoxigenine-labelled probe for the E1A viral genome. A hybridisation mixture containing labelled PBR322 (an unrelated adenovirus probe) served as a control. The probe DNA for the E1A gene was a 756 base pair fragment of the E1A region of adenovirus type 5 which had been subcloned into pUC13 (gift of JC Hogg, Vancouver, BC, Canada). ${ }^{6}$ The 